\title{
Effect of Relative Amplitude on Bridge Deck Flutter
}

\author{
Xin Zhang ${ }^{1}$, James Mark William Brownjohn ${ }^{2}$ \\ School of CEE, Nanyang Technological University, 50 Nanyang Avenue, \\ Singapore, 639798, Singapore
}

\begin{abstract}
:
Self-excited wind forces on a bridge deck can be nonlinear even when the vibration amplitude of the body is small. This phenomenon is evaluated in this paper. Experiments detecting the nonlinearity are performed first, with the concept of "relative amplitude", i.e. the amplitude of the externally triggered free vibration relative to the envelope of the ambient response of an elastically supported rigid sectional model. Two types of sectional model, a twin-deck bluff model (model A) and a partially streamlined box girder model (model B) are tested with two extreme cases of relative amplitude. Based on the flutter derivatives of model $\mathrm{B}$, a flutter boundary prediction is subsequently carried out on a cable-supported bridge to manifest the changes of critical flutter wind velocity due to different relative amplitudes. The effect of relative amplitude on flutter derivatives and on the flutter boundary reveals, from the structural point of view, a complex relationship between the self-excited forces and the "structural vibration noise" due to ambient wind forces. Although the aeroelastic forces are linear when the body motion due to an external trigger is not affected significantly by ambient wind forces, they are nonlinear when the noise component in the vibration cannot be neglected.
\end{abstract}

Keywords: Flutter, Relative Amplitude, Ambient Wind Excitation and Sectional Model Testing

\footnotetext{
${ }^{1}$ Research Fellow, Correspondence Author, School of CEE, Nanyang Technological University 50 Nanyang Avenue, Singapore, 639798, Singapore. Email: cxzhang@ntu.edu.sg

${ }^{2}$ Associate Professor, School of CEE, Nanyang Technological University. Email: cjames@ntu.edu.sg
} 


\section{Introduction}

In the formulation of self-excited wind forces on cable-supported bridge decks, a linearized flutter derivative model is often used [1]. The amplitude dependency of the flutter derivatives has been indicated by previous researches [2][3]. From their points of view, nonlinearity does not exist for the small amplitude case. As a matter of fact, however, the self-excited wind forces due to a pulse response of the deck can be nonlinear even if the response amplitude is small.

Historical study [1] showed that the flutter derivative presentation of the interactive forces holds strictly for sinusoidal oscillation or exponentially modified sinusoidal motion of decay rates less than $20 \%$. Therefore, for a flexible bridge in the wind, the flutter analysis predicts the critical wind speed for a smooth sustained sinusoidal motion of the deck, which indicates the bridge is going to lose its stability due to negative damping.

A "smooth" sinusoidal motion is rarely the real case when there are disturbances in the aerodynamic forces. An initially small amplitude sinusoidal motion can only be considered as a "noisy" sinusoidal one. It needs to be answered in the first place whether or not the noisy sinusoidal motion will grow in the "environmental noise" and become larger in amplitude making itself smoother (the ambient vibration then becomes relatively unimportant) before a flutter prediction is applied.

It is necessary, in this case, to investigate how the interactive force changes due to the effect of the "noise" in the structural motion. The changes in the interactive forces will be indicated by a change in the flutter derivatives.

\section{The Relative Amplitude Effect}

We shall limit our discussions to an elastically supported rigid sectional bridge model subjected to a pulse input to trigger the model to vibrate. The interactive forces under investigation are the self-excited wind loads generated by the pulse response of the rigid body. In all the cases discussed below, the absolute values of vibration amplitude are small. 
The relative amplitude is then defined as the triggered vibration amplitude of the model relative to "structural noise" in the vibration due to the ambient wind excitation. To quantify it, we define the relative amplitude as $R_{a}=A_{0} / \Delta$ in the simulation of Figure 1 , where $A_{0}$ is the mechanically triggered vibration amplitude and $\Delta$ is a characteristic measurement representing the ambient vibration magnitude. Relative amplitude is thus defined in onedimensional. There is no problem to apply it to a multi-dimensional case.

This study is to manifest that the relative amplitude may affect flutter derivatives and the flutter boundary. This effect reveals, from the structural point of view, a relationship between the self-excited forces and the "structural vibration noise" due to fluctuating ambient wind forces. If $R_{a} \rightarrow \infty$, the triggered vibration is totally smooth. If $R_{a} \rightarrow 0$, the triggered vibration is severely affected by the ambient dynamic wind load. The ambient response exists for either smooth or turbulent flow due to the fact that small fluctuations in the oncoming wind are inevitable and the signature turbulence is an inherent part of bluff body dynamics. The ambient response forms the environment for the impulse response to exist. It could be different stories if the pulse response of the sectional model is strong and affects the surrounding flow, or the pulse response is small with all the ambient properties of the aeroelastic system unchanged.

The difference will be illustrated by the various flutter derivatives identified with different relative amplitudes.

\section{Using Output Covariance as Markov Parameters}

Experiments with the large relative amplitude, i.e. transient vibration testing, are common. It is difficult, if not impossible, to identify directly the flutter derivatives when the pulse response amplitude is smaller than the magnitude of the ambient vibration. However, a nominal pulse response for the ambient vibration can be retrieved mathematically by using output covariance [4][5]. This corresponds to identifying the extreme case where the pulse response amplitude tends to zero. Therefore, in the study of relative amplitude effect, it is 
possible to compare two extreme cases, i.e. large $R_{a}$ transient vibration case and zero $R_{a}$ ambient response case.

Because in current experiments, except for the relative amplitudes, all other experimental conditions remain the same, it is reasonable to state that if the output covariance produces a state matrix considerably different form the physically triggered case, there is a relative amplitude effect on the flutter derivatives.

It is a classical result in stochastic identification that the output covariance can be used as the discrete version of pulse response function, i.e. Markov parameters, of a deterministic linear time invariant system, when the measurement noise in the equipment and process noise (dynamic ambient wind excitations) is white (wide-banded in the practical sense) and zero mean. A proof is presented in the Appendix I.

We assume, in the experiments, the displacement measurement is zero mean. If the deck has a static displacement due to the static wind load, it can be treated as a centered process, i.e.

$$
Y(t)=Y(t)-E[Y(t)]
$$

where $E[\bullet]$ denotes expectation operation.

Therefore, we always assume we are dealing with a zero mean process.

The input term, buffeting forces on the bridge deck model, is random and unknown and assumed to be wide banded noise because a real world process will not be white noise. The approximation is a widely adopted one in the application of system identification techniques as long as the noise frequency band is considerably larger than the system frequencies band. The errors so generated should be tolerable. A typical turbulence power spectral density is shown in Figure 2 at wind speed of $17.5 \mathrm{~m} / \mathrm{s}$. It can be observed that the turbulence is wide banded therefore meet the requirement for the application of the output covariance method.

One consideration is that within the time period of measurement of the ambient vibration, the system is actually non-stationary due to uncontrollable experimental conditions. Therefore, in calculating the output covariance, some numerical consideration has been taken to overcome 
the problem.

The structure function was introduced by Kolmogorov [6] in his study of locally isotropic and homogeneous stochastic turbulence. It is defined as:

$$
Z(\tau)=\lim _{T \rightarrow \infty} \frac{1}{T} \int_{0}^{T}[Y(t)-Y(t+\tau)]^{2} d t=2\left(C_{t}(0)-C_{t}(\tau)\right)
$$

where $C_{t}(\tau)$ is the auto-covariance function of the measurement after time $t+\tau$.

For ambient vibration, the covariance function for zero lag time is constant, and the structure function is the sum of the negative covariance function and a constant. If the measurement shows slow fluctuation or has a time varying trend, i.e. shows marked derivation from a stationary behavior, the method of structure function may be advantageous because the structure function can tolerate more low frequency noise than the correlation function [7].

Figure 3 shows a typical calculated output covariance $C_{i}(k)(k=1,2 \ldots N-1$ is the number of lags) from the ambient response by structure function. It can be observed that the signal is quite smooth and suitable to be used in system identification.

\section{$4 \quad$ Experiments and Results}

Two sectional models (Figure 4,5) were tested. The experimental environment was kept the same for the same model in either ambient and transient vibration testing. The setup is shown in Figure 6. The model was restrained in the lateral direction to allow two-dimensional tests. Eight vertical springs were hung from two frames to hold the sectional model in position. These springs were pre-tensioned to avoid nonlinearity. The stiffness of the springs was chosen to make the model vibrate in a suitable frequency, so that a suitable range of reduced wind speeds can be covered by the experiment. The separations between the springs could be adjusted according to the required rotational frequency. Laser displacement sensors were used to measure the displacement history of the model, with one below the front edge and the other below the rear edge to record the vertical and rotational displacement. A single hot-wire anemometer was mounted in front of the sectional model at mid-span location to record the 
wind speed. The typical turbulence intensity of the oncoming wind is less than $2 \%$, e.g. $1.7 \%$ at wind speed of $17.5 \mathrm{~m} / \mathrm{s}$. Therefore, the wind is considered smooth. The following discussion will be limited to smooth flow case. For turbulent flow cases, more researches are needed.

The experimental procedure for the tests is as follows:

1) One of the bridge deck models, model A or model B, was installed.

2) Lateral DOF was constrained with thin wire for 2DOF experiment. Measurement of the system properties was made with free decaying vibration of the model by giving it an initial displacement when there was no wind.

3) Five-minute ambient response records of the model under the action of wind were recorded.

4) Three to five free decay tests were conducted under the same condition as in step 3) except for the initial conditions.

5) Wind speed was increased. Experiments in steps 3 and 4 were repeated. The wind velocities varied from minimum to maximum wind speed at a reasonable interval.

6) After the wind speed reached the upper limit, the other model replaced the first one. Experiments from 2) to 5) were repeated.

Flutter derivatives were then identified from the transient vibration and the output covariance of the ambient vibration. The identification method used was ERA (eigensystem realization algorithm) [8].

Figures 7-8 show the flutter derivatives vs. reduced wind velocity (RU) of model A and model B identified with transient and ambient vibration. For model B, the quality of $A_{2}^{*}$ identified from ambient case is poor; the dotted line in Figure $8 \mathrm{~b}$ is used as the fitting curve for it.

The features of some of the flutter derivatives are very different for different relative amplitudes, suggesting nonlinearity in the self-excited wind load. In the flutter derivatives for the twin deck bluff model (model A), differences are observed in $H_{2}^{*}, H_{3}^{*}$ and $H_{4}^{*}$. However there is no big difference in $A_{i}^{*},(i=1, \cdots, 4)$. For Model $\mathrm{B}, H_{1}^{*}, H_{2}^{*}$ and $H_{3}^{*}$ are among the most sensitive parameters to the relative amplitude. In the transient vibration, where the value 
of relative amplitude is high, the interaction between the vertical and rotational DOF is strong within lower range of the reduced velocity both in terms of aeroelastic stiffness and damping; while in the ambient response $\left(R_{a}=0\right)$, this effect is reduced greatly. $A_{4}^{*}$ is also sensitive to the relative amplitude.

\section{$5 \quad$ Flutter Analysis Considering Relative Amplitude Effect}

Differences between the flutter derivatives identified from transient and ambient vibration testing suggest further study is needed to investigate the effect of relative amplitude on the flutter boundary of a full bridge.

Figure 9 gives the three-dimensional view and finite element mesh of a bridge with partially streamlined box girder cross-section (section model B). Table 1 summarizes the material properties and other dimensions required in calculation. The main span is $1410 \mathrm{~m}$, with side span of 530 and $280 \mathrm{~m}$. The steel box-sections are $22 \mathrm{~m}$ wide and $4.5 \mathrm{~m}$ deep. The towers are box section, 6 by $6 \mathrm{~m}$ at the bases and 4.5 by 4.5 at the tower tops. To facilitate the inclusion of an aeroelastic load model, 3-D beam elements were used to model the deck structure. Spar elements (having no flexural stiffness) were used to present the main cable and hanger. They have the facility to accommodate the initial strain value. The tower was analyzed using beam elements with tension, compression, torsion and bending capabilities. The modal analysis was conducted by using commercial software, ANSYS. Table 2 summarizes the modal analysis results.

The frequency domain method developed by Jain et al [9] is used to perform the flutter instability analysis, which requires solving an aeroelastically-influenced eigen-problem. The flutter condition is obtained at the reduced frequency satisfying equation

$$
|E(K)|=0
$$

The general term of the impedance matrix $E(K)$ is

$$
E_{i j}=-K^{2} \delta_{i j}+i K A_{i j}(K)+B_{i j}(K)
$$


where $i=\sqrt{-1}$ and $\delta_{i j}$ is the Kronecker delta function defined as:

$$
\delta_{i j}=\left\{\begin{array}{ll}
1 & i=j \\
0 & i \neq j
\end{array} .\right.
$$

$A_{i j}$ and $B_{i j}$ are shown in Appendix II. The nontrivial solution $\bar{\xi}$ of the aeroelastically influenced eigenvalue problem

$$
E \bar{\xi}=0
$$

indicates the relative participation of each structural mode under the flutter condition.

Figure 10 is the analysis result using flutter derivatives from transient vibration testing. This is equivalent to defining two surfaces, one for the real part and the other for the imaginary part of $|E|$. These two surfaces are functions of reduced velocity $R U$ and vibration frequency $f$. The intersection of these surfaces with $R U \sim f$ plane is obtained by linear interpolation. Then the zero contour curves of the real surface (solid line) and imaginary surface (dashed line) are obtained with piecewise linear approximation and their intersections can be determined either numerically or graphically. These intersection points define the flutter conditions.

There are two flutter frequencies identified: $0.27 \mathrm{~Hz}$ and $0.39 \mathrm{~Hz}$, corresponding to flutter wind speed $52.7 \mathrm{~m} / \mathrm{s}$ and $75.9 \mathrm{~m} / \mathrm{s}$ respectively. The flutter modes corresponding to them are shown in Figure 11. The result shows, for flutter derivatives identified from transient vibration, flutter may happen at two different wind speeds with different flutter frequencies and modes. More flutter conditions may exist beyond the reduced velocity range covered by the experiments. However only low reduced velocity solutions are important in the practical sense and are calculated.

For flutter derivatives corresponding to the pulse response of ambient vibration $\left(R_{a}=0\right)$, there is no flutter condition found below wind reduced velocity value of 8 . 
Based on the concept of relative amplitude, there should be two thresholds $U_{\text {Amb }}$ and $U_{\text {Tran }}$ of the wind speed. The former is for a signal to diverge in the context of ambient response, the latter is for a larger amplitude signal to diverge after going outside "ambient vibration envelope”. If a signal cannot diverge in the former case, i.e. $U<U_{A m b}$, it would not go to the stage of latter case. Theoretically, the structure is stable to wind excitation. If the signal diverges from ambient response to larger amplitude but subsequently decays, i.e. $U_{A m b}<U<U_{\text {Tran }}$, the response is bounded. If the signal diverges in both cases, i.e. $U_{A m b}<U \& U_{\text {Tran }}<U$, the system is not stable.

It is interesting to consider the case where $U_{\text {Tran }}<U_{A m b}$. Under this condition, the judgment of the onset of flutter based on $U_{A m b}$ is not safe, since there could be other sources of excitation contributing to the dynamic response. A strong gust, for example, could push the bridge deck outside the normal ambient vibration envelope. After the gust, the bridge deck may oscillate back from outside into the ambient vibration envelope. Before the gust, the critical wind speed for flutter is $U_{a m b}$, after the gust, however, the critical wind speed may change to $U_{\text {tran }}$, which may be lower than $U_{a m b}$. This is shown in Figure 12. If flutter happens in this way, a transient rather than steady critical flutter wind speed exists. It will be conservative to use $U_{\text {Tran }}$ as the design flutter wind speed in this case.

\section{Summary}

In this study, nonlinearity in self-excited wind forces is detected through the concept of relative amplitude. By comparing the flutter derivatives identified from triggered free decay and output covariance of ambient vibration of elastically supported rigid sectional bridge models, the relative amplitude effect on the interactive wind forces is manifested. This effect, from the structural point of view, reveals a complex relationship between the self-excited forces and the "structural vibration noise" due to ambient wind excitations. Although the aeroelastic forces are linear when the body motion due to an external trigger is not affected significantly by the turbulence, they are nonlinear when the noise component in the vibration due to the turbulence is not negligible. The experiments were conducted in smooth flow; more experiments are needed to have a discussion on the relative amplitude effect under turbulent 
condition. Conclusions apply to the specific sectional type tested in the study.

Flutter derivatives of the partially streamlined box girder section identified from transient and ambient vibration testing were used to manifest the relative amplitude effect on flutter boundary. The flutter derivatives from ambient vibration were found aeroelastically stable, while the flutter derivatives from transient vibration were not. Therefore, as far as this section type is concerned, the relative amplitude effect appears to have a stabilizing consequence. 


\section{Appendix I Output Covariance}

(Overschee and Moor [10])

When the measurement noise $v$ in the equipment and process noise $p$ (ambient wind forces) is white and zero mean, then

$$
E\left[X \cdot q^{T}\right]=0 \text { and } E\left[X \cdot w^{T}\right]=0
$$

where $q(i)=B p(i)$ and $w(i)=D v(i) . B$ and $D$ are input and feed through matrix of a state space model respectively.

The Lyapunov equation for the state covariance matrix is

$$
\Sigma_{i+1}^{s}=E\left[X(i+1) \cdot X^{T}(i+1)\right]=A \Sigma_{i}^{s} A^{T}+Q
$$

where $Q=E\left[q(i) \cdot q^{T}(i)\right]$ and $A$ is the state matrix.

If output covariance is defined as

$$
C_{i}(k)=E\left[Y(i+k) \cdot Y^{T}(i)\right]
$$

then

$$
C_{i}(0)=E\left[Y(i) \cdot Y^{T}(i)\right]=C \Sigma_{i}^{s} C^{T}+R
$$

where $R=E\left[w(i) \cdot w^{T}(i)\right]$

If define

$$
G=E\left[X(i+1) \cdot Y^{T}(i)\right]=A \Sigma_{i}^{s} C^{T}+S,
$$

where $S=E[q(i) \cdot w(i)]$, we have for $k=1,2, \cdots$

$$
C_{i}(k)=C A^{k-1} G
$$

This produces a new state-space model of $A, G, C, C_{i}(0)$. 


\section{Appendix II Coefficients of the Impedance Matrix Elements}

(Jain et al [9])

The coefficients of the impedance matrix element $E_{i j}$ are

$$
\begin{aligned}
A_{i j}(k)=2 \zeta_{i} K_{i} \delta_{i j} & \frac{\rho B_{d}^{4} l K}{2 I_{i}}\left[H_{1}^{*} G_{h_{i} h_{j}}+H_{2}^{*} G_{h_{i} \alpha_{j}}+H_{5}^{*} G_{h_{i} p_{j}}+P_{1}^{*} G_{p_{i} p_{j}}\right. \\
& \left.+P_{2}^{*} G_{p_{i} \alpha_{j}}+P_{5}^{*} G_{p_{i} h_{j}}+A_{1}^{*} G_{\alpha_{i} h_{j}}+A_{2}^{*} G_{\alpha_{i} \alpha_{j}}+A_{5}^{*} G_{\alpha_{i} p_{j}}\right] \\
B_{i j}(k)=K_{i}^{2} \delta_{i j}- & \frac{\rho B_{d}^{4} l K^{2}}{2 I_{i}}\left[H_{3}^{*} G_{h_{i} \alpha_{j}}+H_{4}^{*} G_{h_{i} h_{j}}+H_{6}^{*} G_{h_{i} p_{j}}+P_{3}^{*} G_{p_{i} \alpha_{j}}\right. \\
& \left.+P_{4}^{*} G_{p_{i} p_{j}}+P_{6}^{*} G_{p_{i} h_{j}}+A_{3}^{*} G_{\alpha_{i} \alpha_{j}}+A_{4}^{*} G_{\alpha_{i} h_{j}}+A_{6}^{*} G_{\alpha_{i} p_{j}}\right]
\end{aligned}
$$

where $K=B_{d} \omega / U$ is the reduced frequency and $K_{i}=B_{d} \omega_{i} / U$ is the reduced frequency of

mode $i, H_{m}^{*}, A_{m}^{*}, P_{m}^{*},(m=1, \cdots, 6)$ are flutter derivatives and the modal integrals $G_{r_{i} s_{j}}$ are obtained by integration over the deck, which is the primarily aerodynamic load source

$$
G_{r_{i} s_{j}}=\int_{0}^{l} r_{i}(x) s_{j}(x) \frac{d x}{l}
$$

where $r_{i}=h_{i}, p_{i}$ or $\alpha_{i} ; s_{j}=h_{j}, p_{j}$ or $\alpha_{j}$. 


\section{Reference}

[1] Scanlan, R.H. and Tomko, J. J. (1971)

Airfoil and Bridge Deck Flutter Derivatives

Journal of Engineering Mechanics, ASCE 97(6) p1717-1737

[2] Scanlan, R. H. (1997)

Amplitude and Turbulence Effects on Bridge Flutter Derivatives

Journal of Structural Engineering, ACSE, 123(2), p232-236

[3] Falco, M; Curami, A and Zasso, A. (1992)

Nonlinear Effects in Sectional Model Aeroelastic Parameters Identification

Journal of Wind Engineering and Industrial Aerodynamics, 41-44 (1992) p1321-1332

[4] Hoen, C.; Moan, N. and Remseth, S. (1993)

System Identification of Structures Exposed to Environmental Loads

Structural Dynamics-EURODYN'93, Moan Et Al. (eds) Balkema, Rotterdam p835843

[5] Jakobsen, J. B. and Hansen, E.; (1995)

Determination of Aerodynamic Derivatives by a System Identification Method Journal of Wind Engineering and Industrial Aerodynamics, 57 (1995) p295-305

[6] Kolmogorov, A. N., (1941)

Local Structure of Turbulence in Non-Compressible Flow with High Reynolds Numbers

Dokl. Acad Sci., USSR, 30, p301-305

[7] Solnes, J. (1997)

Stochastic Processes and Random Vibration Theory and Practice

John Wiley \& Sons, 1997

[8] Juang, J. N. (1994)

Applied System Identification

PTR Prentice-Hall, Inc. Englewood Cliffs, New Jersey

[9] Jain, A; Jones, N. P. and Scanlan, R. H. (1996)

Coupled Flutter and Buffeting Analysis of Long-Span Bridges

Journal of Structural Engineering, ASCE, 122(7), p716-725

[10] Overschee, P.V. and Moor, B.D. (1996)

Subspace Identification for Linear Systems, Theory- Implementation-Application

Kluwer Academic Publishers 


\section{List of Tables}

Table 1 Material Properties of the Bridge

Table 2 Dynamic Properties of the Bridge

\section{List of Figures}

Figure 1 Definition of Relative Amplitude

Figure 2 Power Spectral Density of Lateral Turbulence U=17.5m/s

Figure 3 A Typical Output Covariance

Figure 4 Model A: Twin Deck Bluff Model (Dimension in mm)

Figure 5 Model B: Streamlined Box Girder Model (Dimension mm)

Figure 6 Setup for Free Vibration Test (One End)

Figure 7a 2DOF H (Model A)

Figure $7 \mathrm{~b}$ 2DOF A (Model A)

Figure 8a 2DOF H (Model B)

Figure 8b 2DOF A (Model B)

Figure 9 Plot of the Bridge

Figure 10 E-Matrix (Transient Vibration Case)

Figure 11a First Flutter Mode

Figure 11b Second Flutter Mode

Figure 12 Non-Stationary Flutter Boundary 


\section{List of Symbols}

A State matrix of discrete state space model

$A_{0} \quad$ Mechanically triggered vibration amplitude

$A_{i j}(K), B_{i j}(K)$ Variables in $E_{i j}$

$B \quad$ Input matrix of discrete state space model

$B_{d} \quad$ Width of the Bridge Deck

C Output matrix

$C_{i}(k)$ Output covariance

D Feed through matrix

$E[\bullet] \quad$ Expectation operator

E Impedance matrix

$E_{i j} \quad$ Element in impedance matrix

$G \quad$ Input matrix for covariance dynamics

$G_{r_{i} s_{j}} \quad$ Modal integral

$h, p, \alpha$ Displacements of the rigid body in vertical, lateral and rotational direction, respectively

$h_{i}, p_{i}, \alpha_{i} i^{\text {th }}$ Vertical, lateral and rotational Modes

$H_{i}^{*}, A_{i}^{*}, P_{i}^{*}(i=1, \cdots, 6)$ Flutter Derivatives

$I_{i} \quad$ Generalized inertia

K Reduced Frequency

$l \quad$ Bridge deck length

$p(t) \quad$ Buffeting force

$R_{a} \quad$ Relative amplitude

$U \quad$ Wind speed

$X \quad$ State vector

$Y \quad$ Displacement

$Z(\tau)$ Structural function

$\sum_{i+1}^{s} \quad$ Variable

$v(t) \quad$ Measurement noise

$\tau \quad$ Time 
$\omega, \omega_{i} \quad$ Circular frequency

$\bar{\xi} \quad$ Participation factor vector of structural modes at flutter

$\delta_{i j} \quad$ Kronecker delta function

$\Delta \quad$ Characteristic measurement representing the ambient vibration magnitude 
Table 1 Material Properties of the Bridge

\begin{tabular}{|c|c|c|}
\hline \multicolumn{2}{|r|}{ Cable } & \\
\hline \multicolumn{2}{|c|}{ Young's modulus of cables } & $193 \mathrm{KN} / \mathrm{mm}^{2}$ \\
\hline \multicolumn{2}{|c|}{ Young's modulus of hangers } & $140 \mathrm{KN} / \mathrm{mm}^{2}$ \\
\hline \multicolumn{2}{|l|}{ Area per hanger } & $0.0021 \mathrm{~m}^{2}$ \\
\hline \multirow{2}{*}{ Area of each cable } & Main Span & $0.29 \mathrm{~m}^{2}$ \\
\hline & Side Span & $0.31 \mathrm{~m}^{2}$ \\
\hline \multicolumn{3}{|l|}{ Box Girder Deck } \\
\hline \multicolumn{2}{|l|}{ Young’s Modulus } & $200 \mathrm{KN} / \mathrm{mm}^{2}$ \\
\hline \multicolumn{2}{|l|}{ Axial area of steal } & $0.73 \mathrm{~m}^{2}$ \\
\hline \multicolumn{2}{|c|}{ Second moment of area for vertical bending } & $1.940 \mathrm{~m}^{4}$ \\
\hline \multicolumn{2}{|c|}{ Second moment of area for lateral bending } & $37.07 \mathrm{~m}^{4}$ \\
\hline \multicolumn{2}{|l|}{ Torsional rigidity } & $4.5 \mathrm{~m}^{4}$ \\
\hline \multicolumn{3}{|l|}{ Towers } \\
\hline \multicolumn{2}{|l|}{ Young's modulus } & $20 \mathrm{KN} / \mathrm{mm}^{2}$ \\
\hline \multicolumn{2}{|c|}{ Average axial material area of each leg } & $20.37 \mathrm{~m}^{2}$ \\
\hline \multicolumn{2}{|c|}{ Average second moment of area of each tower leg for longitudinal bending } & $66.8 \mathrm{~m}^{4}$ \\
\hline \multicolumn{2}{|c|}{ Average second moment of area of each tower leg for lateral bending } & $68.24 \mathrm{~m}^{4}$ \\
\hline \multicolumn{2}{|c|}{ Average torsional rigidity of each tower leg } & $113.1 \mathrm{~m}^{4}$ \\
\hline
\end{tabular}


Table 2 Dynamic Properties of the Bridge

\begin{tabular}{c|c|c}
\hline \hline \multirow{2}{*}{ Mode No. } & Mode Type & Frequency \\
& & $n_{i}(\mathrm{~Hz})$ \\
\hline \hline 1 & $\mathrm{~L}, 1^{\text {st }} \mathrm{S}$ & 0.0688 \\
\hline 2 & $\mathrm{~V}, 1^{\text {st }} \mathrm{S}$ & 0.1277 \\
\hline 3 & $\mathrm{~L}, 1^{\text {st }} \mathrm{AS}$ & 0.1591 \\
\hline 4 & $\mathrm{~V}, 1^{\text {st }} \mathrm{AS}$ & 0.1646 \\
\hline 5 & $\mathrm{~V}, 2^{\text {nd }} \mathrm{S}$ & 0.1897 \\
\hline 13 & $\mathrm{~V}, 2^{\text {nd }} \mathrm{AS}$ & 0.2498 \\
\hline 14 & $\mathrm{LT}, \mathrm{L}, 2^{\text {nd }} \mathrm{S} ; \mathrm{T}, 1^{\text {st }} \mathrm{S}$ & 0.2816 \\
\hline 16 & $\mathrm{~V}, 3^{\text {rd }} \mathrm{S}$ & 0.3246 \\
\hline 26 & $\mathrm{~V}, 3^{\text {rd }} \mathrm{AS}$ & 0.4022 \\
\hline 28 & $\mathrm{~T}, 1^{\text {st }} \mathrm{AS}$ & 0.45853 \\
\hline \hline
\end{tabular}

Note: S=symmetrical; AS=anti-symmetrical; L: Lateral; V=vertical; LT=lateral-torsion; T=torsion and

Structural modes used in flutter boundary computation are normalized with respect to mass matrix. 


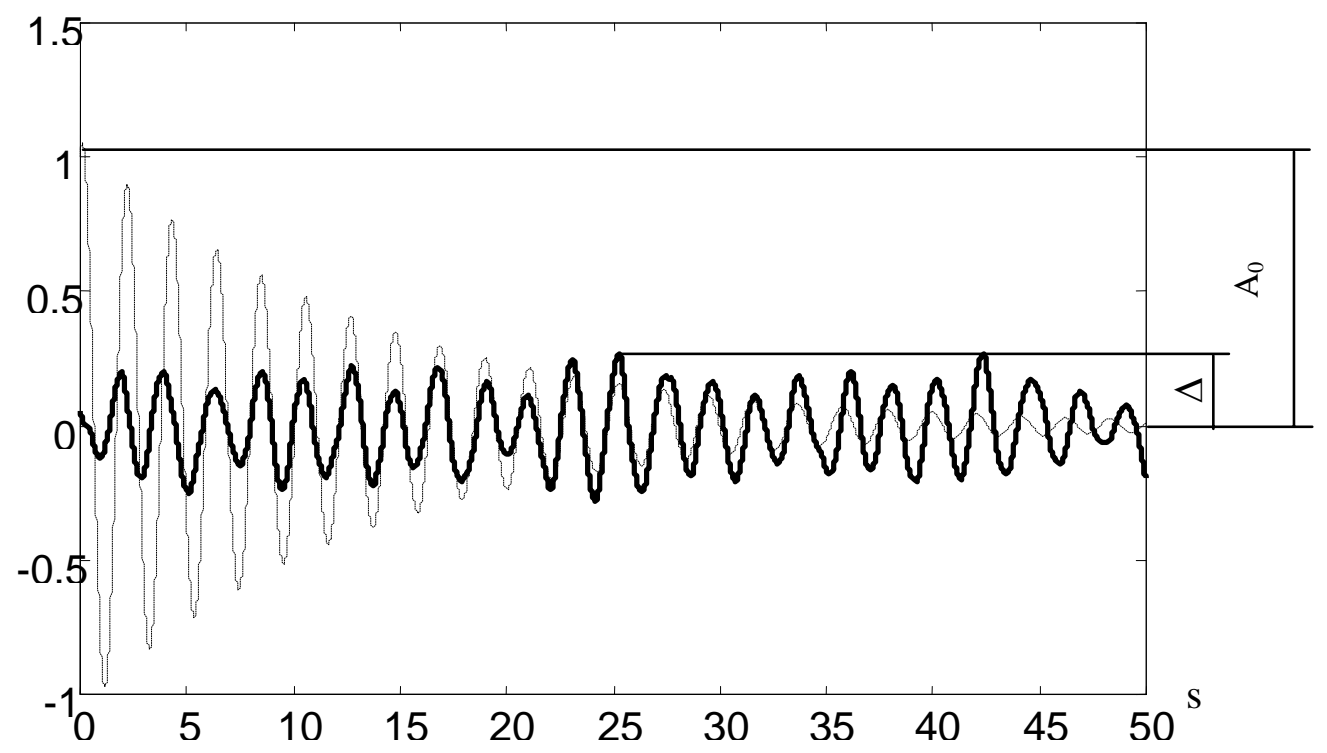

Figure 1 Definition of Relative Amplitude

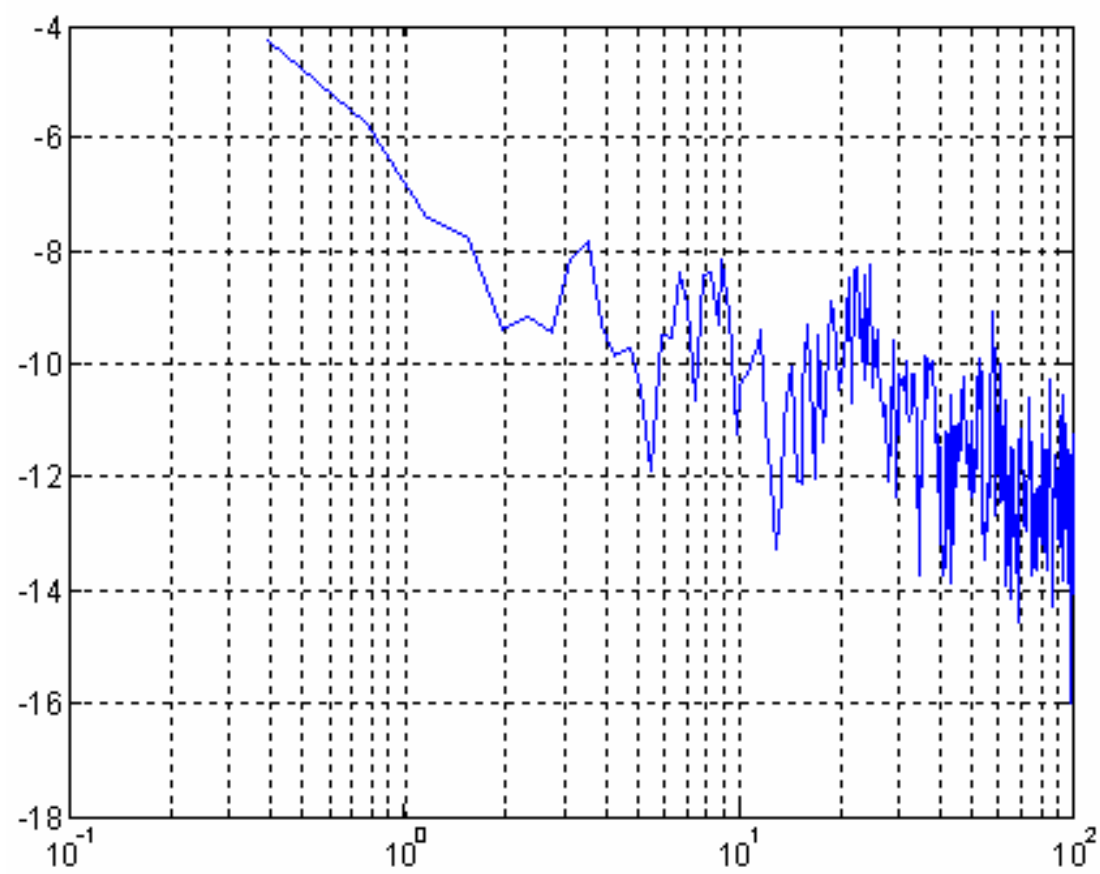

Figure 2 Power Spectral Density of Lateral Turbulence U=17.5m/s 

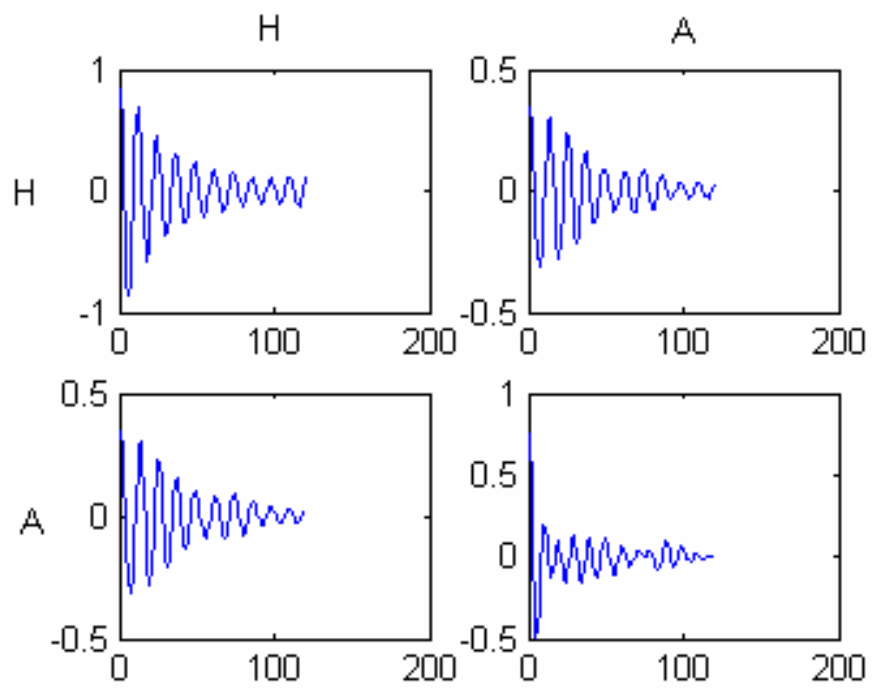

Figure 3 A Typical Output Covariance 


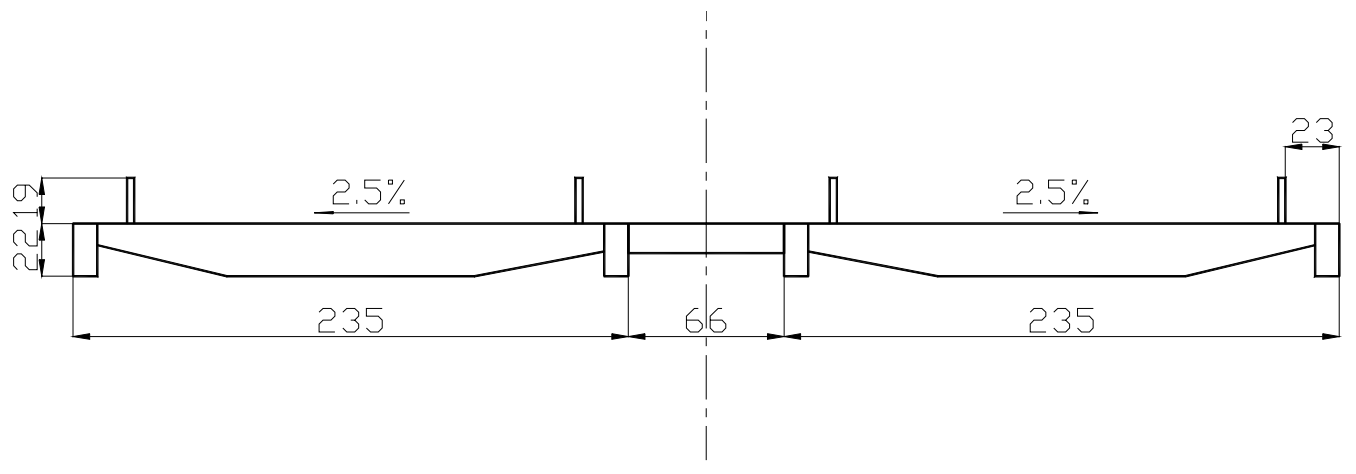

Figure 4 Model A: Twin Deck Bluff Model (Dimension in mm) 


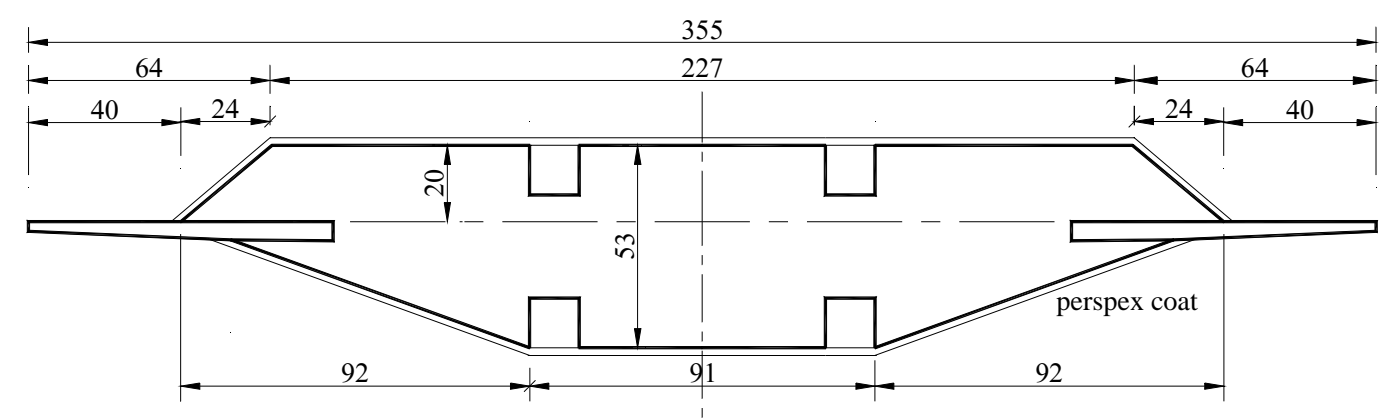

Figure 5 Model B: Streamlined Box Girder Model (Dimension mm) 


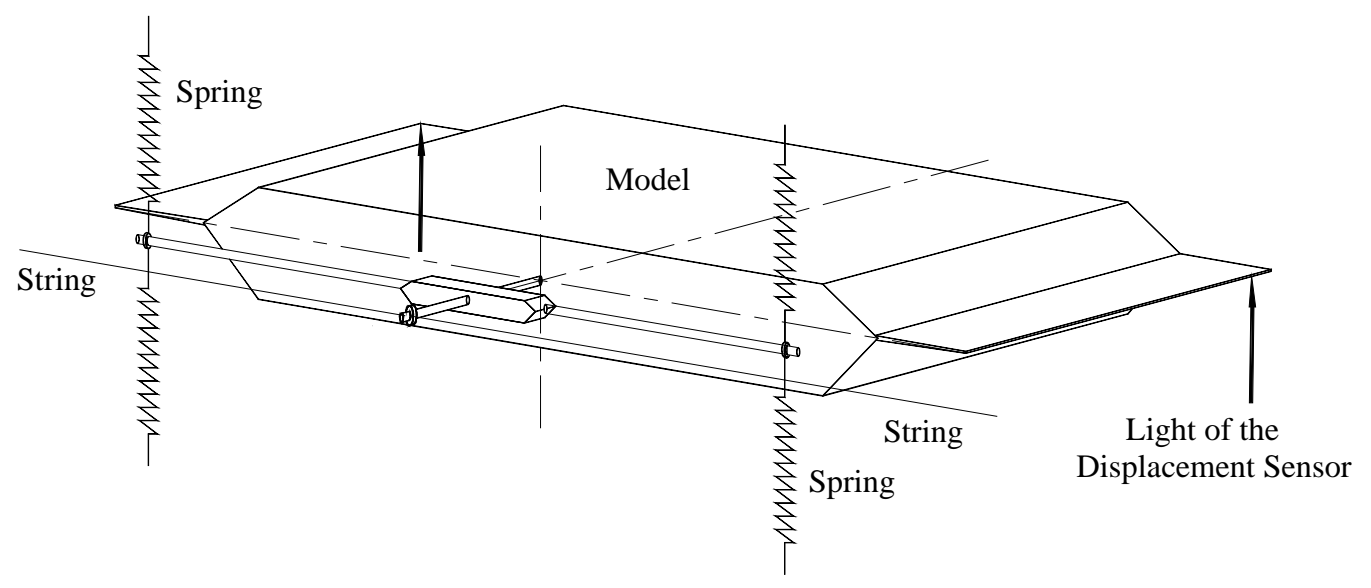

Figure 6 Setup for Free Vibration Test (One End) 

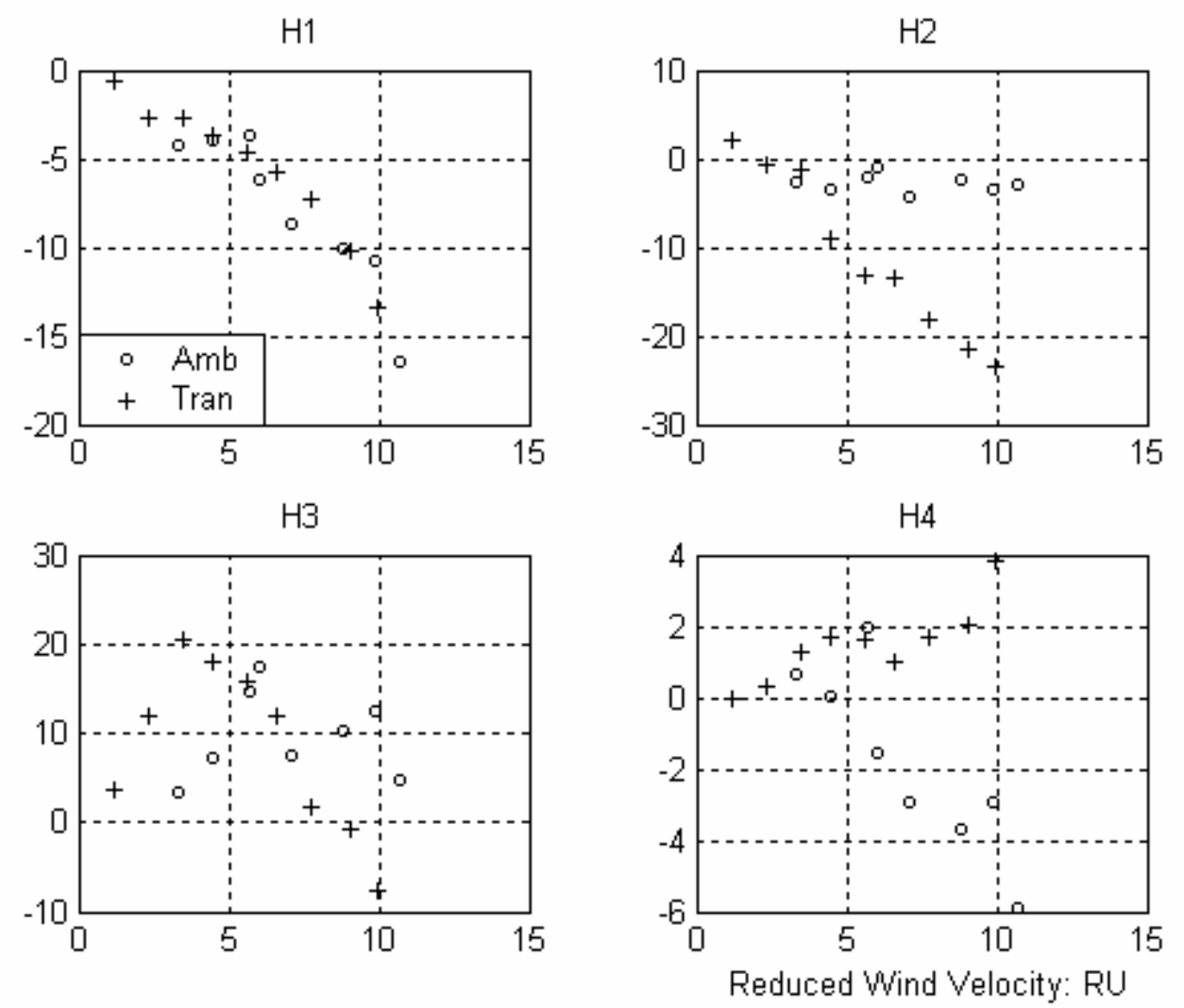

Figure 7a 2DOF H (Model A) 

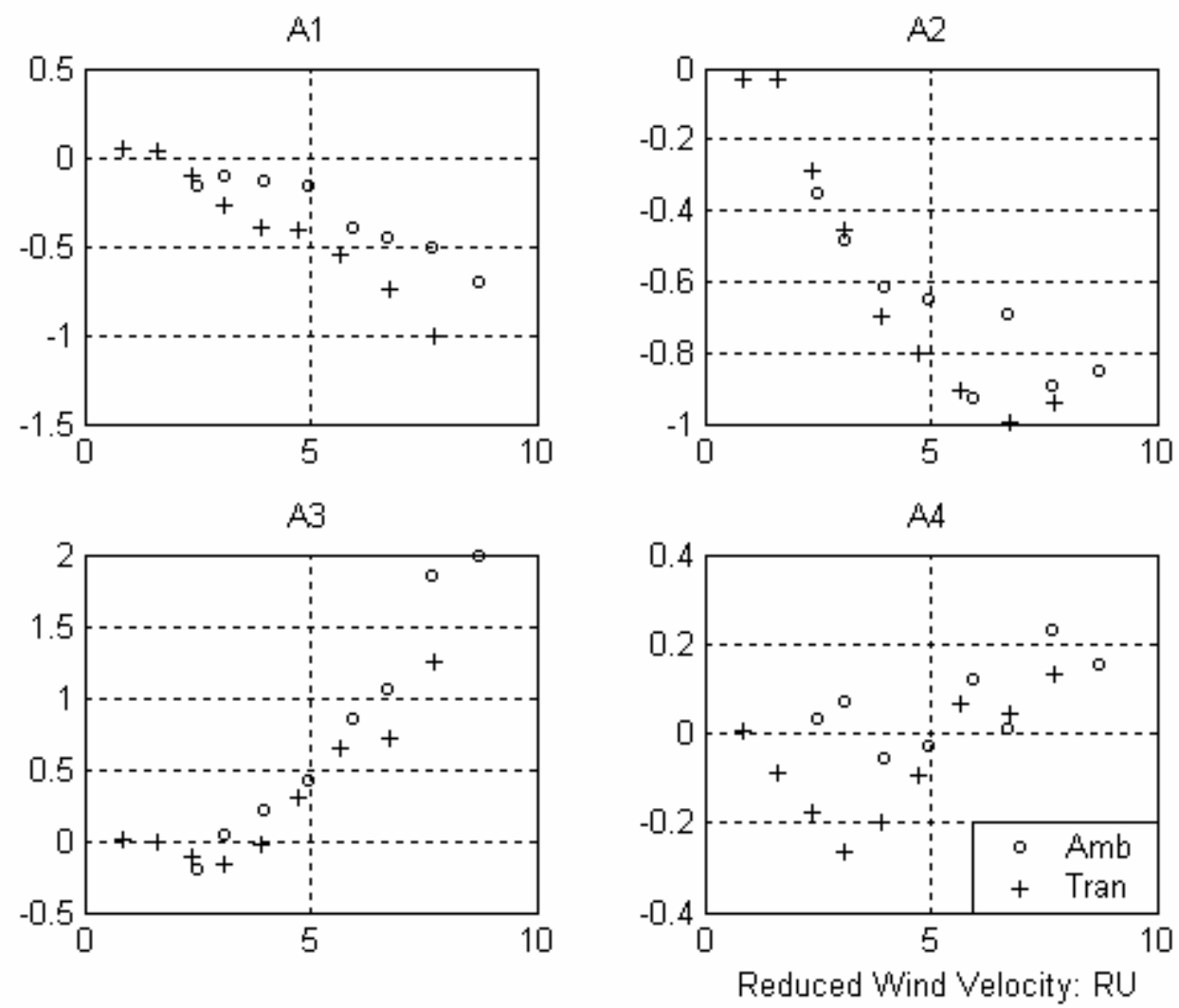

Figure 7b 2DOF A (Model A) 

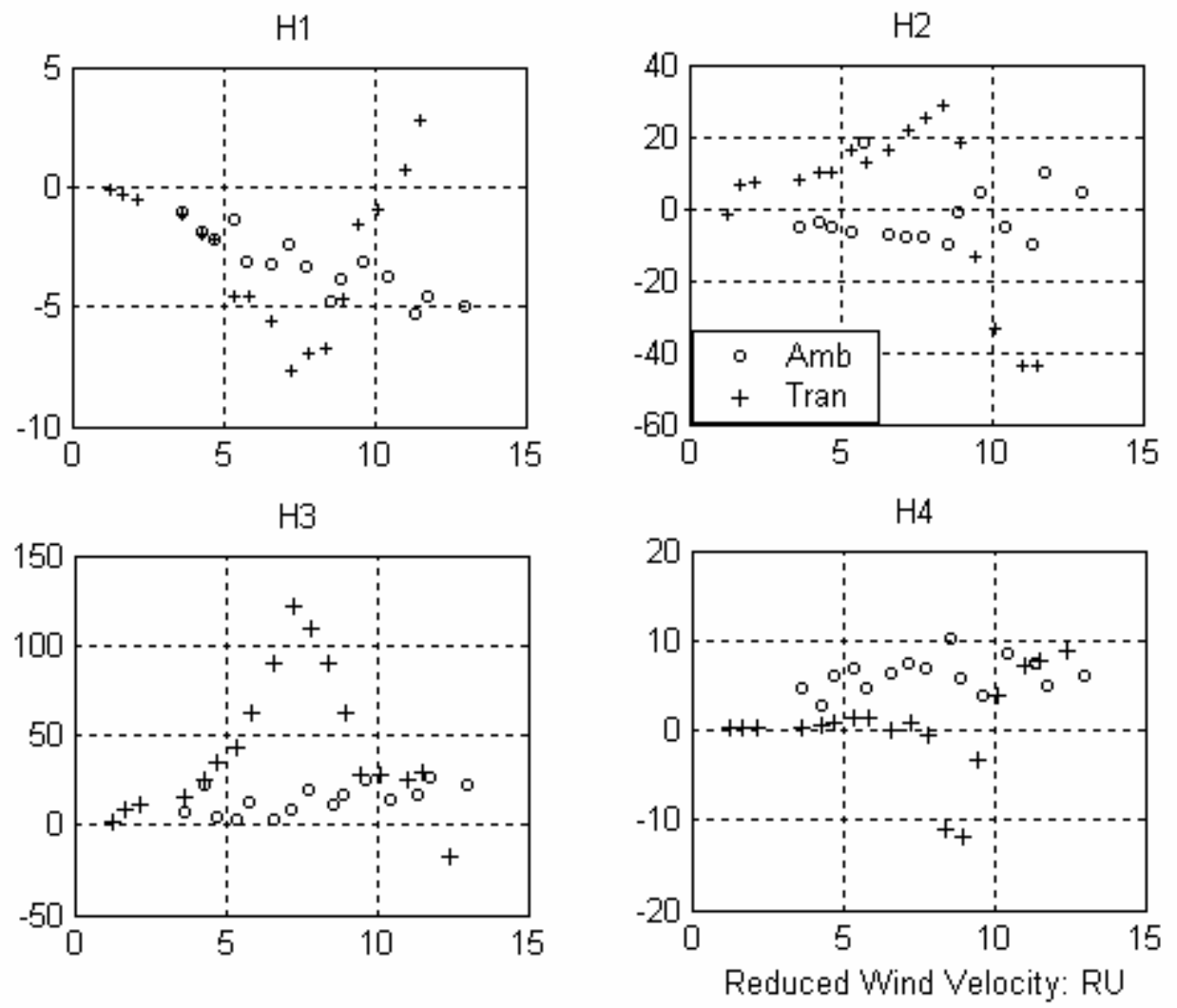

Figure 8a 2DOF H (Model B) 

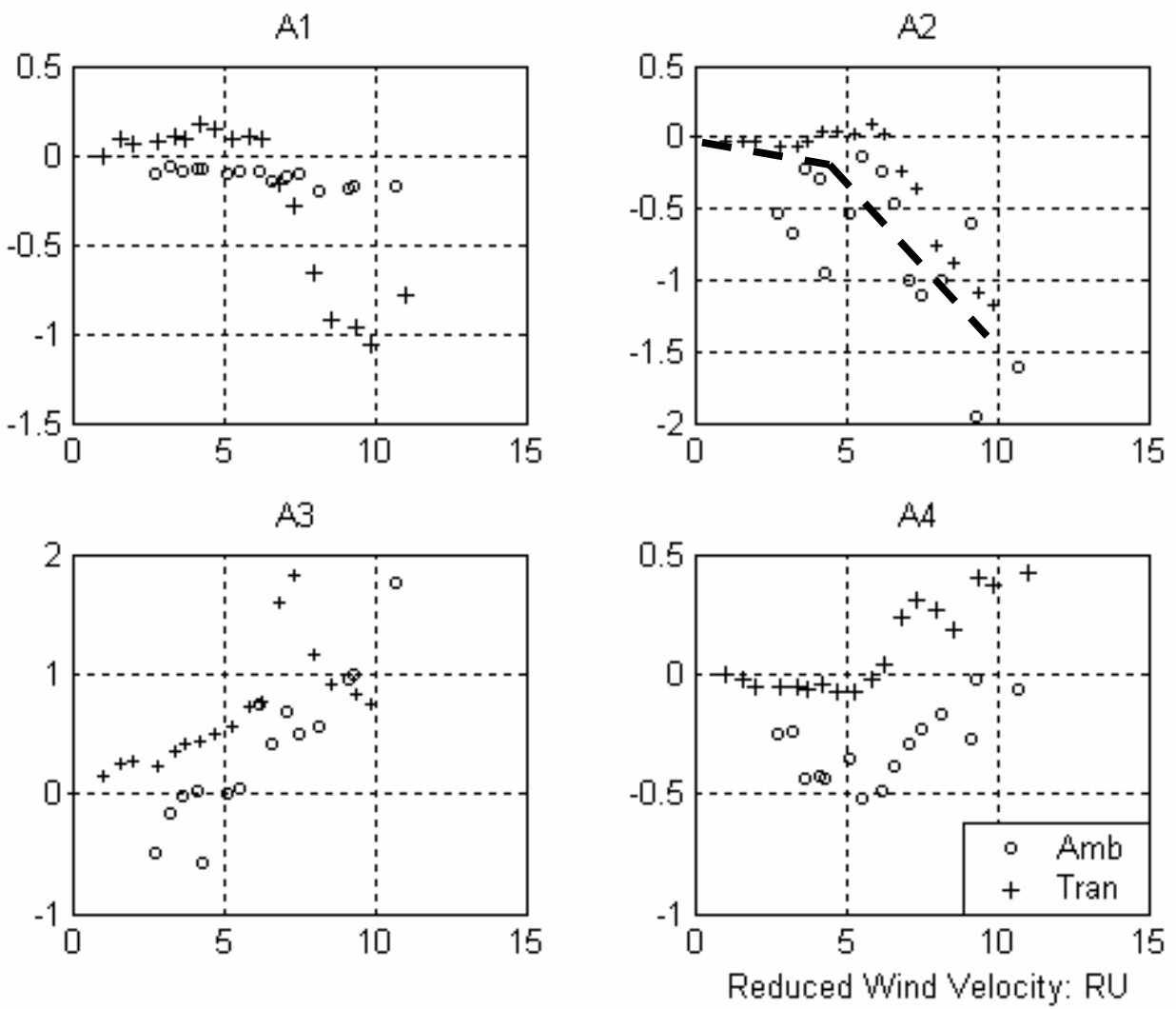

Figure 8b 2DOF A (Model B) 


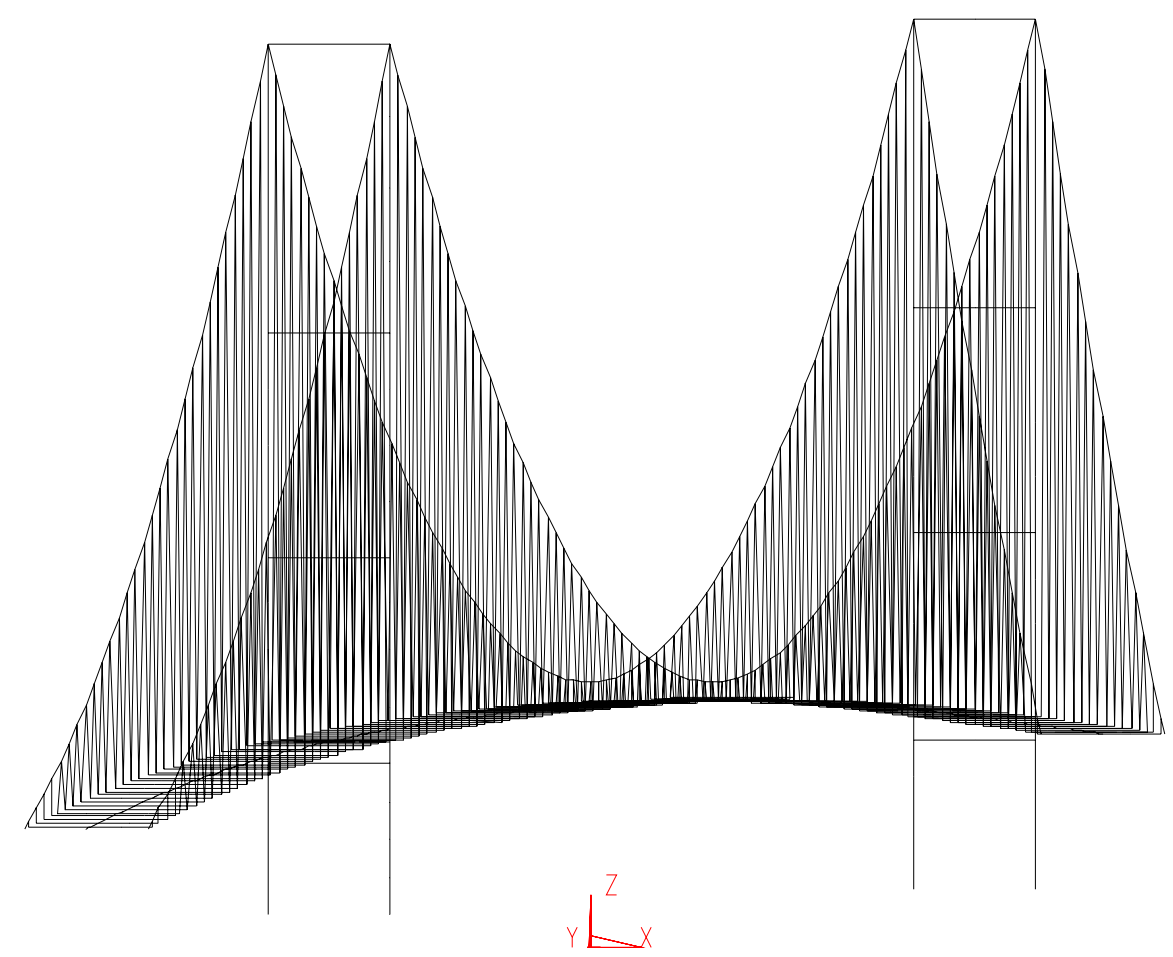

Figure 9 Plot of the Bridge 


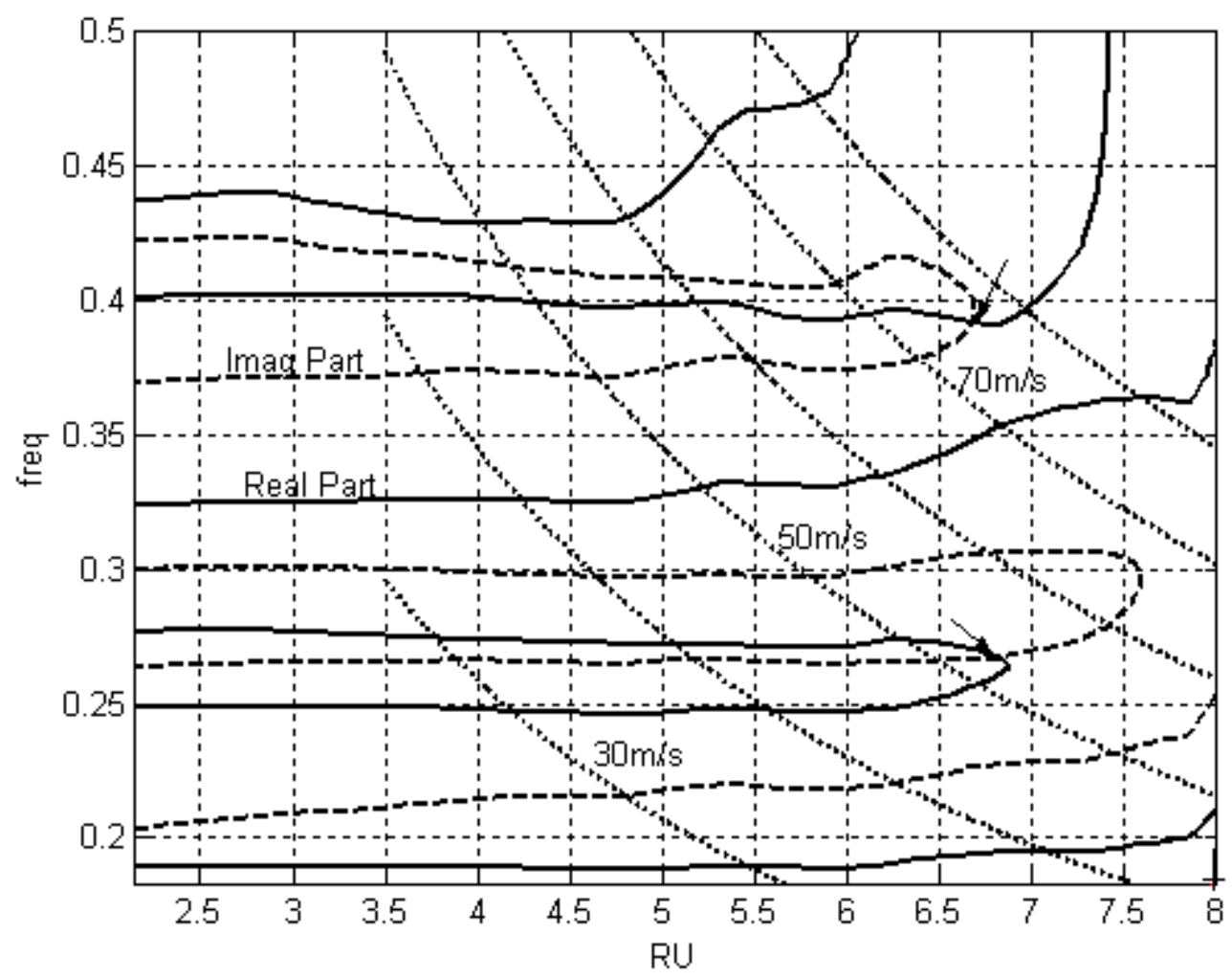

Figure 10 E Matrix (Transient Vibration Case) 

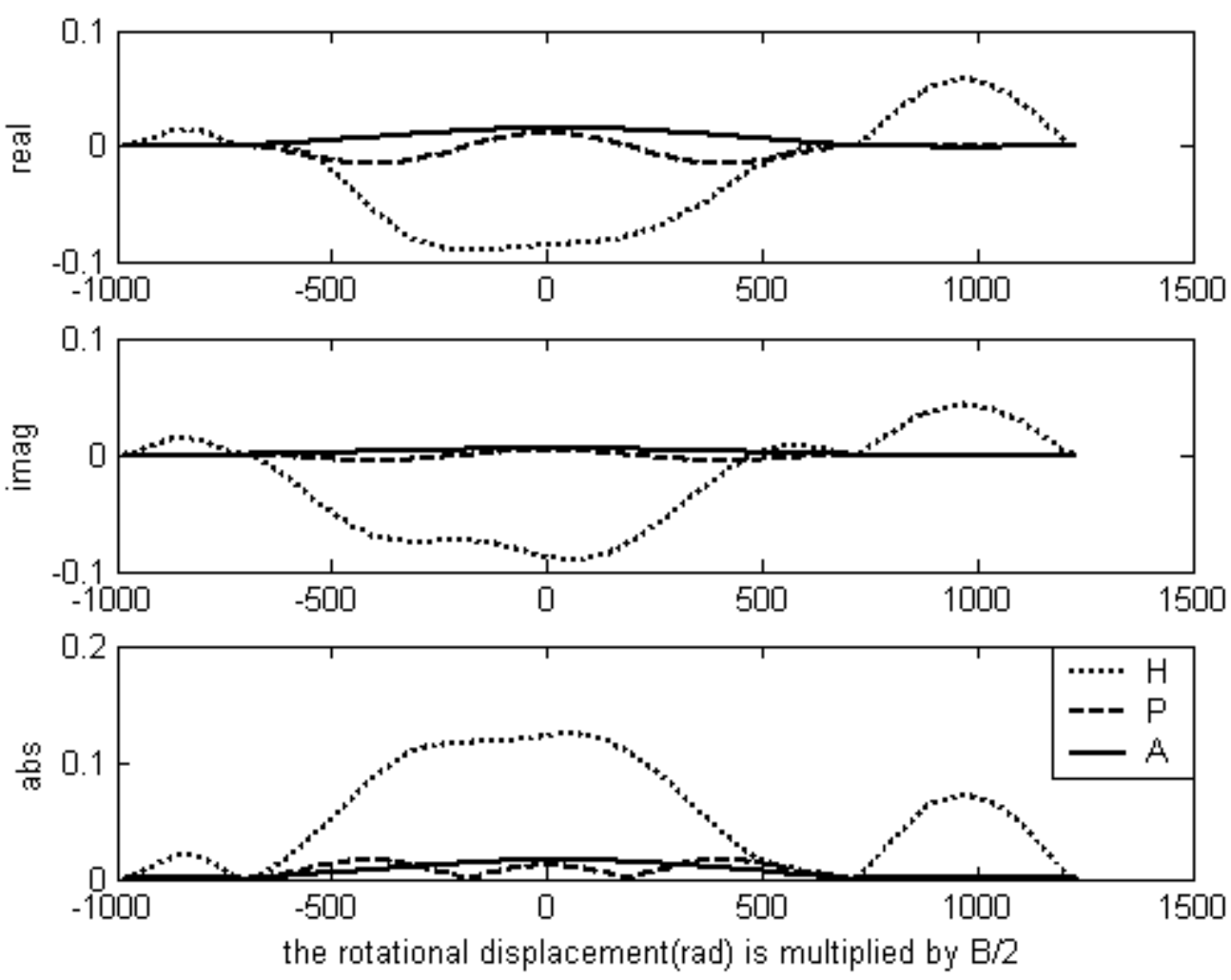

Figure 11a First Flutter Mode 

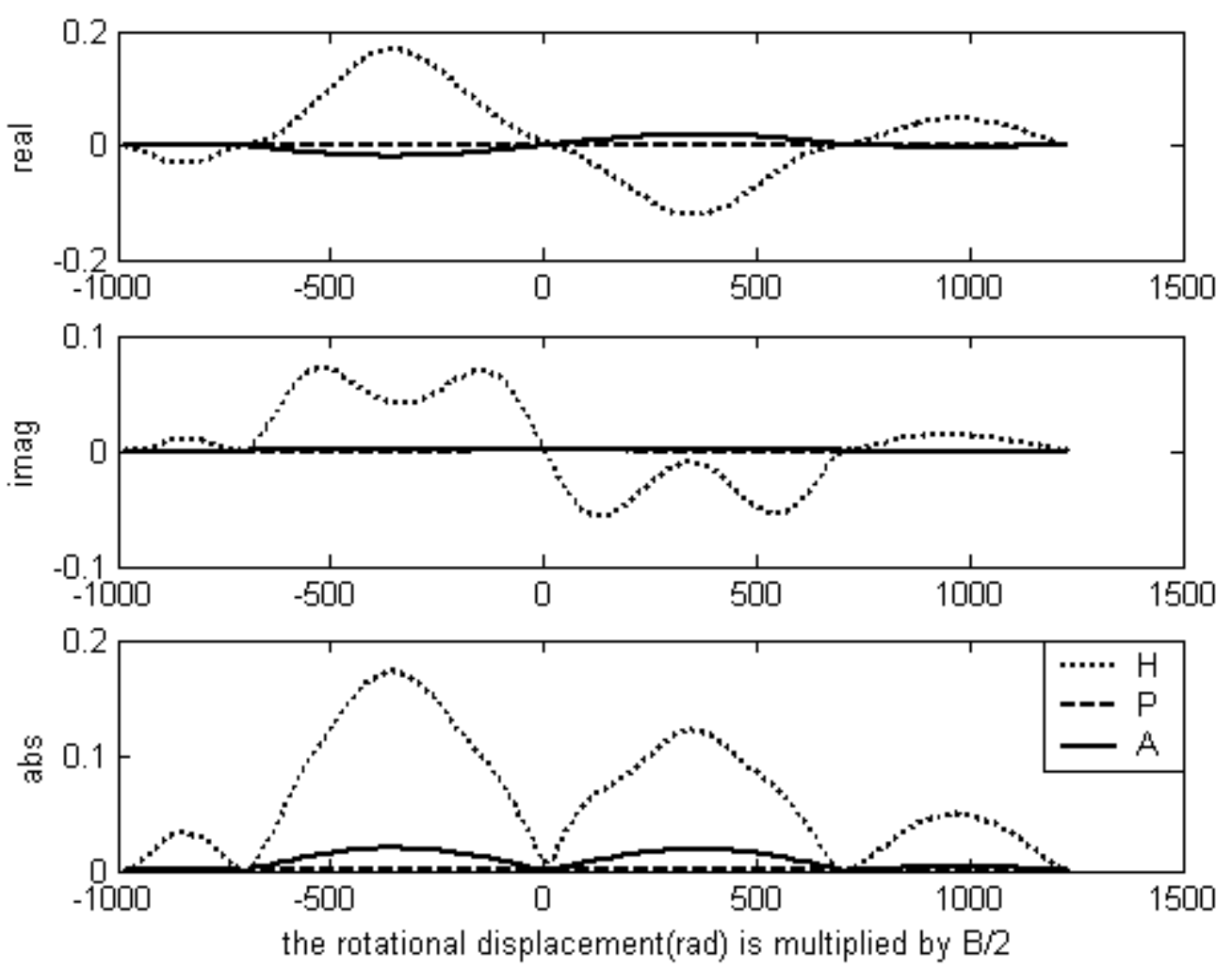

Figure 11b Second Flutter Mode 


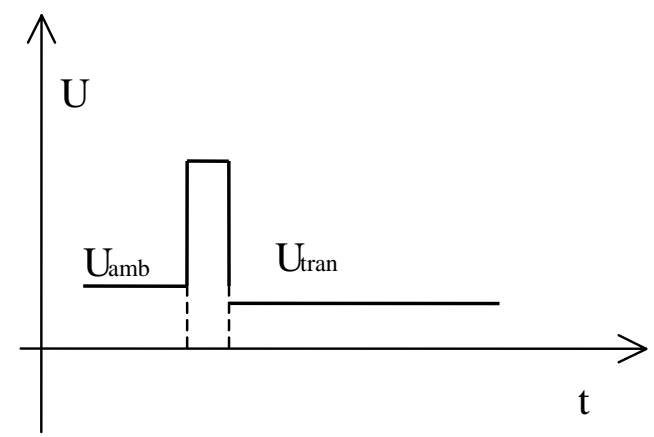

Figure 12 Non-Stationary Flutter Boundary 